\title{
HE4 - not only an ovarian cancer biomarker - a brief review
}

\author{
Marta Maksimiuk1, Michał P. Budzik², Andrzej Deptała², Anna M. Badowska-Kozakiewicz²
}

'Students' Scientific Organization of Cancer Cell Biology, Department of Cancer Prevention, Medical University of Warsaw, Poland

${ }^{2}$ Department of Cancer Prevention, Medical University of Warsaw, Poland

Human epididymis protein 4 (HE4) was firstly identified in epididymal epithelial cells and described as a protease inhibitor playing a role in spermatogenesis. Regarding numerous studies proving its diverse potential as a prognostic and predictive factor in ovarian cancer, it was incorporated into ROMA algorithm. Nevertheless, recent studies have shown that serum level of HE4 is not exclusive to ovarian cancer. As a result, doctors using ROMA algorithm for stratifying patients with ovarian cancer must be aware of other conditions that may affect serum level of HE4. This review comprises different conditions connected with high level of HE4 that might impact ovarian cancer diagnosing process. Moreover, discovering increased HE4 level in various conditions should open discussion about its applicability in diseases other than ovarian cancer.

NOWOTWORY J Oncol 2019; 69, 3-4: 142-145

Key words: human epididymis protein 4, ovarian cancer, ROMA algorithm, biomarkers

\section{Introduction}

Human epididymis protein 4 (HE4) was firstly identified in epididymal epithelial cells and described as a protease inhibitor playing role in spermatogenesis. It is encoded by WAP 4-disulfide core domain 2 (WFDC2) [1]. Selective overexpression of HE4 has a well-established role in ovarian cancer tumorigenesis, but little is known about its possible role in other conditions [2]. Regarding numerous studies proving its diverse potential as a prognostic and predictive factor in ovarian cancer, it was incorporated into ROMA algorithm, that is a quantitative test based on the serum level of HE4, CA125, and combined with menopausal status [1]. Using this algorithm allows for stratifying patients into two groups - in a high or low ovarian cancer risk and as a result avoiding unnecessary surgeries [3, 4].

On the other hand, recent studies have shown that serum level of HE4 is not exclusive to ovarian cancer [5-8]. Described conditions comprise both cancer and other diseases. The most numerous group of researches about HE4 as a possible new biomarker is connected with lung cancer, especially non-small cell lung cancer (NSCLC) $[9,10]$. Some authors even suggest that HE4 secretion might play an extensive role in NSCLC progression, like in ovarian cancer [7]. Another conditions suggested to be connected with HE4 comprise chronic kidney disease [5], renal fibrosis [11], cancers of intestinal tract [6, 12, 13], breast cancer [8] and heart failure [14].

Due to the fact that, as mentioned above, the increased level of HE4 is detectable not only in patients with ovarian cancer, but also in patients with other diseases, doctors using ROMA algorithm for stratifying patients with ovarian cancer must be aware of other conditions that may affect the serum level of HE4. The main aim of this review was to gather and discuss diseases connected with HE4, described in the literature. According to our best knowledge, a similar review encompassing different possible directions in HE4 usage have not been published yet.

\section{Kidney}

HE4 was proven to be expressed in the distal convoluted tubules of the kidney [15] and since then scientists try to find out correlation between its expression and the occurrence of chosen conditions. Chronic kidney disease (CKD) is told to become a worldwide public health problem with mean global prevalence at $13.4 \%$. Owing to the fact that detecting CKD at early stages allows its appropriate management there 
is an ongoing need for searching new diagnostic indicators [16]. One of the most recently suggested is HE4. In the study conducted by Yuan et al. (2017) [5] serum level of HE4 increased significantly with renal function decline and was proven to achieve better diagnostic ability, sensitivity and specificity than other laboratory indicators, so as a result, it was suggested as the strongest predictor for CKD. What is more, increased levels of HE4 were detectable even at early stages of CKD. HE4 level in blood samples derived from patients with renal disfunction was positively correlated with level of creatinine, urea and cystatin $C$ as far as acute and chronic renal dysfunctions are concerned and has higher diagnostic value with 100\% specificity and sensitivity [17]. On the other hand, various studies proved inverse correlation between HE4 and eGFR with statistical significance $[11,17]$.

The most common final pathological way of CKD is renal fibrosis. HE4 has been recently reported as one of the mediators of this phenomenon because of inhibiting the degradation of type I collagen [18]. In the study conducted by Wan et al. (2016) [11] higher levels of HE4 were detected in patients with more severe renal fibrosis and significant correlation between HE4 and degree of renal fibrosis was observed. ROC curve analysis pointed out HE4 as a suitable biomarker for the diagnosis of renal fibrosis. Moreover, HE4 is suggested as a biomarker for predicting renal fibrosis in kidney transplant recipients due to its increased level and correlation with the severity of the disease [19].

Lupus nephritis (LN) is a common manifestation of systemic lupus erythematosus (SLE) and a major cause of morbidity and mortality in these patients. Increased serum HE4 level was proved to be associated with development of LN in SLE patients in two independent studies $[20,21]$. However, detailed mechanism leading to development of LN with HE4 contribution remains unclear and needs further research.

\section{Intestinal tract}

Gastric cancer (GC), despite its decreasing incidence ratio, has still low 5-year survival rate, thus needs factors indicating its prognosis and improving treatment [22]. The first investigation concerning HE4 expression in GC was performed by Guo et al. (2015) [6]. They discovered upregulation of this glycoprotein in gastric cancer tissues and significant correlation with Lauren classification, TNM stage and tumor size. Silencing HE4 inhibited proliferation, migration and enhanced apoptosis in studied tissues. Regarding these discoveries, HE4 might play an important role in progression of GC and become a new target for treatment. Another aspect of HE4 in GC concerns sensitivity to radiotherapy. Peng et al. (2019) [23] pointed out hypoxia-induced upregulation of HE4 as a reason for resistance to radiotherapy due to the fact that stable knockdown of HE4 sensitized cancer cells and xenograft tumors to radiotherapy. As a conclusion, radiotherapy connected with HE4 knockdown might become a potential therapeutic aim in GC.
Next example of association between resistance to radiotherapy and expression of HE4 is colorectal cancer (CRC). Shi et al. (2018) [12] demonstrated that WFDC2 deficiency improved the radiation resistance in CRC. miR-149 - a small noncoding RNA regulating post-transcriptional gene expression was proved to inhibit HE4 expression in CRC cells and sensitize CRC to radiation both in vivo and in vitro. As a result, exogenous administration of miR-149 mimic combined with radiotherapy might become a new therapeutic promise in CRC. In 2017, Kemal et al. [24] detected high level of HE4 in CRC samples in comparison to healthy controls. They also proposed a HE4 as a new biomarker for stage III-IV CRC due to its significantly positive expression especially in this group. However, the study group was relatively small and without any follow-up information. What is more, CRC seems to present wide range of tumor markers used for diagnosing and staging thus searching new biomarkers might be questioned.

HE4 expression was suggested as a marker of early stage of pancreatic adenocarcinoma. In the study by Huang et al. (2015) [13] serum HE4 levels reached sensitivity of $45.9 \%$ and specificity of $93.6 \%$ with cutoff set at $4.59 \mathrm{ng} / \mathrm{mL}$. Interestingly, combination of HE4 with CA19-9 increased sensitivity to $83.3 \%$ and the combined HE4 and CA $15-3$ to sensitivity of $87.5 \%$ thus set consisting of HE4, CA19-9 and CA15-3 might become a new powerful biomarker panel for early detection of pancreatic adenocarcinoma and diagnostic improvement. Lu et al. (2016) [25] research determined that treatment of recombinant HE4 on pancreatic cancer Suit-2 cell caused significant cells growth, increased DNA synthesis and cell viability in comparison to control group without HE4 treatment. Moreover, treatment with HE4 upregulated PCNA (key molecule for DNA synthesis) and downregulated p21 (a critical cell cycle regulator). To conclude, $\mathrm{HE} 4$ presents an undeniable role in pancreatic cancer development and might be used as a potential biomarker in its early stage detection.

\section{Lung cancer}

Owing to the fact that lung cancer is still a leading cause of cancer morbidity and mortality all over the world, accurate and early diagnostic tools encompassing this malignancy are in the area of scientists' interest [22]. Serum HE4 levels were proven to be significantly higher in NSCLC patients than in benign lung diseases and healthy controls. What is more, its higher level was correlated with high TNM stage, positive lymph nodes metastasis and weight loss [7, 26]. Connecting these facts with documented shorter overall survival (OS) in the group with higher level of HE4 allows us to conclude that serum levels of HE4 might predict poor prognosis in NSCLC patients. Moreover, Mo et al. (2018) [26] observed that HE4 was a satisfying discriminator of lung adenocarcinoma. Although Celik et al. (2017) [27] doubted the reliability of HE4 as a lung cancer biomarker, they admitted that it was a promising candidate for adenocarcinoma treatment. On the 
other hand, Korkmaz et al. (2018) [28] suggested a panel of three tumor markers including HE4 for discriminating lung cancer from benign lung lesions and subtyping as small cell lung cancer (SCLC).

Survivors of lung cancer are at high risk of disease recurrence, thus we are in a need of sensitive methods for their postoperative monitoring. Current monitoring system is based on clinical examination and imaging methods. However, this combination might in some cases turn out to be insufficient. Muley et al. (2019) [29] suggested algorithm of serial serum measurements consisting of HE4 and another biomarker CYFRA 21-1 for a recurrence detection. Their suggestion was based on the observation of 31 out of 115 patients suffering from adenocarcinoma recurrence - serum levels of CYFRA 21-2 and HE4 were significantly higher in samples taken from patients with recurrence in comparison to these derived from patients in remission.

\section{Breast cancer}

Breast cancer (BC) is the most commonly diagnosed cancer among women all over the world [22]. Regarding to this fact, contemporary science tries to find out new markers for more effective diagnosis and treatment because the sensitivity and specificity of known biomarkers such as CA15-3 and CEA are rather low [30]. Gunduz et al. (2016) [8] identified a significant elevation of serum HE4 in comparison to healthy control group and a correlation between the levels of HE4 and CA15-3 in patients suffering from BC. On the basis of these results they proposed HE4 as a potential biomarker for BC. Nevertheless, the presented study was conducted on a small group of patients and authors did not achieve any significant results as far as many well-known clinicopathological factors in BC are concerned, thus it seems to be too early to call a HE4 a potential biomarker in this condition. In recent years miRNA has become a new target in cancer research due to its correlation with prognosis, clinical staging and metastases [31]. Lu et al. (2017) [32] decided to combine plasma miR-127-3p and HE4 in BC analysis. They concluded that levels of both plasma miR-127-3p and HE4 were increased in BC and combined detection greatly improved methods of early diagnosis of $B C$ with sensitivity of $87.4 \%$

\section{Heart failure}

Although new research suggests HE4 as a potential novel biomarker for heart failure (HF), some obstacles may complicate its usage in monitoring of these patients [33]. Most of them are mentioned above - increased level of HE4 is detectable not only in patients with $\mathrm{HF}$, but also in patients with ovarian cancer [2], CKD [5] or pancreatic adenocarcinoma [13]. However, HE4 as a part of multi-marker model, might be a potential aim in the HF stratification [33]. In the study conducted among 567 patients with HF, those with higher HE4 serum level had an unfavorable clinic profile comprising older age, higher NYHA class, greater number of comorbidities such as atrial fibrillation. Plasma HE4 levels were correlated with numerous HF plasma markers (including NT-proBNP, BNP and galectine-3) with the strongest correlation including GDF15 - an emerging prognostic biomarker of cardiovascular diseases [34]. Similar results were achieved by Piek et al. (2017) [14] - strong correlation especially comprising HF severity based on NYHA and NT-proBNP levels was detected. Levels of HE4 was also correlated with risk factors including age, male sex, hypertension and diabetes. What is more, patients with HE4 levels above the median had worse survival rate. Nevertheless, the study was conducted on a relatively small sample size and, as mentioned above, using HE4 as a marker for HF diagnosing and risk stratification might turn out to be impossible due to frequent coincidence between $\mathrm{HF}$ and other conditions which makes HE4 non-specific.

\section{Conclusions}

HE4 is not exclusive for ovarian cancer. In recent studies it was proven to have a possible role in diagnosing other conditions, including cancers. Nevertheless, the connection between its biology, genetics and pathological condition remains unclear. The most important conclusion from this review suggests carefulness while using HE4 in ovarian cancer diagnosing and remembrance about other conditions that might affect our judgement. Furthermore, owing to developing branch of using HE4 in other diseases we might ask ourselves the question about its applicability in yet unknown syndromes.

\section{Conflict of interests: none declared}

\section{Marta Maksimiuk \\ Medical University of Warsaw \\ Department of Cancer Prevention \\ Students' Scientific Organization of Cancer Cell Biology \\ ul. Żwirki i Wigury 81 \\ 02-091 Warszawa, Poland \\ e-mail:marcioszka.m@gmail.com}

\section{Received: 4 Jun 2019}

Accepted: $22 \mathrm{Jul} 2019$

\section{References}

1. Chudecka-Glaz AM. ROMA, an algorithm for ovarian cancer. Clin Chim Acta. 2015; 440: 143-151.

2. Moore RG, Hill EK, Horan T et al. HE4 (WFDC2) gene overexpression promotes ovarian tumor growth. Sci Rep. 2014; 4: 3574.

3. Wei SU, Li H, Zhang B. The diagnostic value of serum HE4 and CA-125 and ROMA index in ovarian cancer. Biomed Rep. 2016; 5 (1): 41-44.

4. Li QL, Wang CJ, Qi P et al. Correlation of preoperative ROMA scores with clinical stage in epithelial ovarian cancer patients. Clin Transl Oncol. 2017; 19 (10): 1260-1267.

5. Yuan T, Li Y. Human epididymis protein 4 as a potential biomarker of vhronic kidney disease in female patients with normal ovarian function. Lab Med. 2017; 48 (3):2 38-243.

6. Guo YD, Wang JH, Lu H et al. The human epididymis protein 4 acts as a prognostic factor and promotes progression of gastric cancer. Tumour Biol. 2015; 36 (4): 2457-2464.

7. Lamy PJ, Plassot C, Pujol JL. Serum HE4: an independent prognostic factor in non-small cell lung cancer. PLoS One. 2015; 10 (6): e0128836. 
8. Gunduz UR, Gunaldi M, Isiksacan N et al. A new marker for breast cancer diagnosis, human epididymis protein 4: A preliminary study. Mol Clin Oncol. 2016; 5 (2): 355-360.

9. Zhong $\mathrm{H}$, Qian $\mathrm{Y}$, Fang $\mathrm{S}$ et al. HE4 expression in lung cancer, a meta-analysis. Clin Chim Acta. 2017; 470: 109-114.

10. Cheng $D$, Sun $Y, H e H$. The diagnostic accuracy of HE4 in lung cancer: a meta-analysis. Dis Markers. 2015; 2015: 352670.

11. Wan J, Wang Y, Cai G et al. Elevated serum concentrations of HE4 as a novel biomarker of disease severity and renal fibrosis in kidney disease. Oncotarget. 2016; 7 (42): 67748-67759.

12. Shi LP, Guo HL, Su YB et al. MicroRNA-149 sensitizes colorectal cancer to radiotherapy by downregulating human epididymis protein 4. Am J Cancer Res. 2018; 8 (1): 30-38.

13. Huang T, Jiang SW, Qin L et al. Expression and diagnostic value of HE4 in pancreatic adenocarcinoma. Int J Mol Sci. 2015; 16 (2): 2956-2970.

14. Piek A, Meijers WC, Schroten NF et al. HE4 serum levels are associated with heart failure severity in patients with chronic heart failure. J Card Fail. 2017; 23 (1): 12-19.

15. Galgano MT, Hampton GM, Frierson HF Jr. Comprehensive analysis of HE4 expression in normal and malignant human tissues. Mod Pathol. 2006; 19 (6): 847-853.

16. Girndt M. [Diagnosis and treatment of chronic kidney disease]. Internist (Berl). 2017; 58 (3): 243-256.

17. Wang L, Sun Y, Cai $X$ et al. The diagnostic value of human epididymis protein 4 as a novel biomarker in patients with renal dysfunction. Int Urol Nephrol. 2018; 50 (11): 2043-2048.

18. Chen P, Yang Q, Li X et al. Potential association between elevated serum human epididymis protein 4 and renal fibrosis: A systemic review and meta-analysis. Medicine (Baltimore). 2017; 96 (36): e7824.

19. Luo J, Wang F, Wan J et al. Serum human epididymis secretory protein 4 as a potential biomarker of renal fibrosis in kidney transplantation recipients. Clin Chim Acta. 2018; 483: 216-221.

20. Ren Y, Xie J, Lin F et al. Serum human epididymis protein 4 is a predictor for developing nephritis in patients with systemic lupus erythematosus: A prospective cohort study. Int Immunopharmacol. 2018;60: 189-193.

21. Yang Z, Zhang Z, Qin B et al. Human epididymis protein 4: a novel biomarker for lupus nephritis and chronic kidney disease in systemic lupus erythematosus. J Clin Lab Anal. 2016; 30 (6): 897-904.
22. Siegel RL, Miller KD, Jemal A. Cancer Statistics, 2017. CA Cancer J Clin. 2017; 67 (1): 7-30.

23. Peng C, Liu G, Huang K et al. Hypoxia-induced upregulation of HE4 is responsible for resistance to radiation therapy of gastric cancer. $\mathrm{Mol}$ Ther Oncolytics. 2019; 12: 49-55.

24. Kemal YN, Demirag GN, Bedir AM et al. Serum human epididymis protein 4 levels in colorectal cancer patients. Mol Clin Oncol. 2017; 7 (3): 481-485

25. Lu Q, Chen H, Senkowski C et al. Recombinant HE4 protein promotes proliferation of pancreatic and endometrial cancer cell lines. Oncol Rep. 2016; 35 (1): 163-170.

26. Mo D, He F. Serum human epididymis secretory protein 4 (HE4) is a potential prognostic biomarker in non-small cell lung cancer. Clin Lab. 2018; 64 (9): 1421-1428.

27. Celik B, Bulut T. Human epididymis protein 4 may not be a reliable screening biomarker for detecting lung carcinoma patients. Biomed Rep. 2017; 7 (4): 297-300.

28. KorkmazET, Koksal D, Aksu F et al. Triple test with tumor markers CYFRA 21.1, HE4, and ProGRP might contribute to diagnosis and subtyping of lung cancer. Clin Biochem. 2018; 58: 15-19.

29. Muley T, He Y, Rolny V et al. Potential for the blood-based biomarkers cytokeratin 19 fragment (CYFRA 21-1) and human epididymal protein 4 (HE4) to detect recurrence during monitoring after surgical resection of adenocarcinoma of the lung. Lung Cancer. 2019; 130: 194-200.

30. Molina R, Barak V, van Dalen A et al. Tumor markers in breast cancer - European Group on Tumor Markers recommendations. Tumour Biol. 2005; 26 (6): 281-293.

31. Guo LJ, Zhang OY. Decreased serum miR-181a is a potential new tool for breast cancer screening. Int J Mol Med. 2012; 30 (3): 680-686.

32. Lu M, Ju S, Shen X et al. Combined detection of plasma miR-127-3p and HE4 improves the diagnostic efficacy of breast cancer. Cancer Biomark. 2017; 18 (2): 143-148.

33. Piek A, Du W, de Boer RA et al. Novel heart failure biomarkers: why do we fail to exploit their potential? Crit Rev Clin Lab Sci. 2018; 55 (4): 246-263.

34. de Boer RA, Cao Q Postmus D et al. The WAP four-disulfide core domain protein HE4: a novel biomarker for heart failure. JACC Heart Fail. 2013; 1 (2): 164-169. 